\title{
THE NORM OF THE SUM OF TWO PROJECTIONS
}

\author{
IVAN VIDAV
}

Abstract. Let $\mathrm{e}$ and $\mathbf{f}, \mathbf{e}+\mathbf{f} \neq 0$, be two projections of a $C^{*}$-algebra $\mathrm{A}$. J. Duncan and P. J. Taylor have shown that $\|\mathbf{e}+\mathbf{f}\|=1+\|\mathbf{e f}\|$. In this paper an algebraic proof of this equality is given.

Let $\mathbf{A}$ be a $C^{*}$-algebra with unit 1 , and let $\mathbf{e}$ and $\mathbf{f}$ be two projections of $\mathbf{A}$, not both zero. J. Duncan and P. J. Taylor have shown [2, Theorem 7] that

$$
\|\mathbf{e}+\mathbf{f}\|=1+\|\mathbf{e f}\| \text {. }
$$

Their proof is geometric. It is based upon a formula of Chandler Davis [1], [3] giving a matrix representation of two projections in generic position in a Hilbert space. Here we shall give an algebraic proof of the above equality.

TheOREM. The spectrum $\sigma(\mathbf{e}+\mathbf{f})$ of $\mathbf{e}+\mathbf{f}$ is contained, with possible exception of the point $\lambda=0$, in the interval $[1-\|\mathbf{e f}\|, 1+\|\mathbf{e f}\|]$. The point $\lambda=1+$ $\|\mathbf{e f}\|$ belongs to $\sigma(\mathbf{e}+\mathbf{f})$. Hence $(1)$ holds.

Proof. Denote by $\mathbf{r}(\mathbf{e f})$ the spectral radius of ef. From the inequalities $(n>1)$

$$
\| \text { efe }\left\|^{n}=\right\|(\text { efe })^{n}\|=\|(\text { ef })^{n} e\|\leqslant\|(\text { ef })^{n}\|=\|(\text { efe })^{n-1} \mathbf{f}\|\leqslant\| \text { efe } \|^{n-1}
$$

we deduce that $\mathbf{r}(\mathrm{ef})=\|\mathbf{e f e}\|=\|\mathbf{e f}\|^{2}$.

Now, let $\lambda$ be any complex number $\neq 0,1$. Since

$$
\begin{aligned}
(\lambda-1+\mathbf{e})(\lambda-\mathbf{e}-\mathbf{f})(\lambda-1+\mathbf{f}) & =[(\lambda-1)(\lambda-\mathbf{f})-\mathbf{e f}](\lambda-1+\mathbf{f}) \\
& =\lambda\left[(\lambda-1)^{2}-\mathbf{e f}\right],
\end{aligned}
$$

we have

$$
\left[(\lambda-1)^{2}-\mathbf{e f}\right]^{-1}=\lambda(\lambda-1+\mathbf{f})^{-1}(\lambda-\mathbf{e}-\mathbf{f})^{-1}(\lambda-1+\mathbf{e})^{-1} .
$$

Hence

$$
\lambda(\lambda-\mathbf{e}-\mathbf{f})^{-1}=(\lambda-1+\mathbf{f})\left[(\lambda-1)^{2}-\mathbf{e f}\right]^{-1}(\lambda-1+\mathbf{e}) .
$$

Because $(\lambda-\mathbf{e})(\lambda-1+\mathbf{e})=\lambda(\lambda-1)$ we get from (2)

$$
\lambda(\lambda-1)^{2}\left[(\lambda-1)^{2}-\mathbf{e f}\right]^{-1}=(\lambda-\mathbf{f})(\lambda-\mathbf{e}-\mathbf{f})^{-1}(\lambda-\mathbf{e}) .
$$

It follows that $(\lambda-\mathbf{e}-\mathbf{f})^{-1}$ exists for $\lambda \neq 0,1$ iff $\left[(\lambda-1)^{2}-\mathbf{e f}\right]^{-1}$ exists.

Received by the editors October 25, 1976 and, in revised form, January 31, 1977. AMS (MOS) subject classifications (1970). Primary 46L05; Secondary 46C05. 
This implies that the spectrum $\sigma(e f)$ of ef is real and belongs to the interval $[0, r(e f)]=\left[0,\|e f\|^{2}\right]$. Let $\lambda \in \sigma(e+f), \lambda \neq 0$. It follows from (2) that $\lambda-$ $1)^{2} \in \sigma(e f) \in\left[0,\|\mathbf{e f}\|^{2}\right]$. Therefore, $1-\|\mathbf{e f}\| \leqslant \lambda \leqslant 1+\|\mathbf{e f}\|$. Since $\lambda=\mathbf{r}(\mathbf{e f})$ $=\|\mathbf{e} f\|^{2} \in \sigma(\mathbf{e f})$, we have $1+\|\mathbf{e f}\| \in \sigma(\mathbf{e}+\mathbf{f})$. Hence, $\|\mathbf{e}+\mathbf{f}\|=1+\|\mathbf{e f}\|$. Q.E.D.

REMARK 1. Let $\lambda \in \sigma(\mathbf{e}+\mathbf{f}), \lambda \neq 0,1,2$. Since $(\lambda-1)^{2} \in \sigma(\mathbf{e f})$, it follows from (3) that $2-\lambda \in \sigma(\mathbf{e}+\mathbf{f})$. Therefore, the set $\sigma(\mathbf{e}+\mathbf{f}) \cap(1-\|\mathbf{e f}\|, 1+$ $\|\mathbf{e f}\|)$ is symmetric with respect to the point $\lambda=1$.

Remark 2. Put $(\lambda-e f)^{-1}=u$ and $(\lambda-e f e)^{-1}=v$. If $v$ exists then ev $=$ ve. From $(\lambda-\mathbf{e f})(1+$ evf $)=\lambda-$ ef $+\mathbf{e}(\lambda-\mathbf{e f e}) \mathbf{v f}=\lambda$ and

$$
(1+\text { evf })(\lambda-\text { ef })=\lambda-\text { ef }+\operatorname{ev}(\lambda-\mathbf{e f e}) \mathbf{f}=\lambda
$$

we have $\lambda u=1+$ evf. Similarly we get $\lambda v=1+$ efue. This implies that $\sigma(e)=\sigma(e f e)$. Further, it follows from (2) and (3) that $\left(\lambda-\mathbf{e}-f^{-1}\right.$ can be expressed by $\left[(\lambda-1)^{2}-e f e\right]^{-1}$, and vice versa.

REMARK 3. If $\|\mathbf{e f}\|<1$, then $\lambda=0$ is an isolated point of $\sigma(\mathbf{e}+\mathbf{f})$ (or possibly $\lambda=0$ belongs to the resolvent set of $e+f)$. In this case the right-hand side of (2) is analytic at $\lambda=0$ with value $(1-\mathbf{f})(1-\mathbf{e f})^{-1}(1-\mathbf{e})$ $=\mathbf{g}$ there. Hence, $\lambda^{-1} \mathbf{g}$ is the principal part of the resolvent $(\lambda-\mathbf{e}-\mathbf{f})^{-1}$ at $\lambda=0$. The coefficient $g$ is the projection of $\mathbf{e}+\mathbf{f}$ corresponding to the isolated point $\lambda=0$ of $\sigma(\mathbf{e}+\mathbf{f})$. If we put $\lambda=2$ in (3) we get $2(1-\mathbf{e f})^{-1}=$ $(2-f)(2-\mathbf{e}-\mathbf{f})^{-1}(2-\mathbf{e})$. Hence we have also

$$
\mathbf{g}=2(1-\mathbf{f})(2-\mathbf{e}-\mathbf{f})^{-1}(1-\mathbf{e}) \text {. }
$$

Suppose now that $\mathbf{e}$ and $\mathbf{f}$ are projections acting in a Hilbert space $\mathbf{H}$. Let $\mathbf{H}_{1}=\mathbf{e H}$ and $\mathbf{H}_{2}=\mathbf{f H}$ be the corresponding base spaces. Then $\mathbf{g} \xi=0, \xi \in$ $\mathbf{H}$, holds if and only if $\mathbf{e} \xi+\mathbf{f} \xi=0$, and $\mathbf{e} \xi+\mathbf{f} \xi=0$ holds if and only if $\mathbf{e} \xi=\mathbf{f} \xi=0$, i.e., $\xi \in \mathbf{H}_{1}^{\perp} \cap \mathbf{H}_{2}^{\perp}$. Hence $\mathbf{g}$ is the projection onto $\mathbf{H}_{1}^{\perp} \cap \mathbf{H}_{2}^{\perp}$. It follows that $2 \mathbf{f}(\mathbf{e}+\mathbf{f})^{-1} \mathrm{e}$ is the projection onto $\mathrm{H}_{1} \cap \mathrm{H}_{2}$, if $(e+f)^{-1}$ exists.

\section{RefERENCES}

1. Chandler Davis, Separation of two linear subspaces, Acta Sci. Math. Szeged 19 (1958), 172-187. MR 20 \#5425.

2. J. Duncan and P. J. Taylor, Norm inequalities for $C^{*}$-algebras, Proc. Royal Soc. Edinburgh 75 (1976), 119-129.

3. P. R. Halmos, Two subspaces, Trans. Amer. Math. Soc. 144 (1969), 381-389. MR 40 \# 4746.

Department of Mathematics, University of Lubluana, Lubluana, Yugoslavia 